Methods Pristane was injected in female $\mathrm{BALB} / \mathrm{c}$ mice to induce the disease. After five months, mice in various groups were treated with prednisone and fed with gluten containing and standard diet for four weeks and applied procedure to detect minor changes in paw swelling, ANA autoantibodies, CCL11, C3c, glucose level and renal damage.

Results We detected increased symptoms of arthritis and gastrointestinal tract involvement in gluten containing diet group compared with standard diet disease control group. ANA autoantibodies, $\mathrm{C} 3 \mathrm{c}$ and renal damage between gluten and standard diet group was non-significant. The remission of SLE manifestations was observed in prednisone treated group except renal damage.

Conclusions From the study it was concluded that gluten intake could worsen the clinical manifestations in SLE patients, therefore, administration of gluten free diet might be a better strategy for SLE patients. However, further confirmatory studies are required in this regard.

\section{SLE RISK HAPLOTYPES ARE ASSOCIATED WITH DEVELOPMENT OF SEROLOGIC AUTOIMMUNITY IN HEALTHY INDIVIDUALS}

${ }^{1} \mathrm{P}$ Raj, ${ }^{2} \mathrm{D}$ Karp*, 'I Dozmorov, 'Q Li, ${ }^{1} \mathrm{E}$ Wakeland. ${ }^{1}$ UT Southwestern Medical Centre, Immunology, Dallas, USA; ${ }^{2}$ UT Southwestern Medical Centre, Rheumatic Diseases Division, Dallas, USA

\subsection{6/lupus-2017-000215.283}

Background and aims ANA are one of the earliest features of lupus, preceding the onset of clinical symptoms. The genetic risk factors that underlie the development of serological autoimmunity are unknown. A genome-wide association study was undertaken to understand the genetics of ANA development.

Methods Serum and DNA were collected from 2635 healthy individuals with no personal history of autoimmunity. Sera from 724 individuals (ANA-, ANA+, and SLE) were assayed by protein microarray quantifying $\operatorname{IgM}$ and $\operatorname{IgG}$ responses to 96 human autoantigens. A nested cohort of subjects consisting of all the ANA+ Caucasian individuals and matched ANAcontrols were genotyped.

Results In healthy individuals, 16.2\% had moderate and 8.0\% had high levels of IgG antinuclear antibodies. ANA+ healthy individuals had a high prevalence of antibodies to non-nuclear and cytoplasmic antigens, while subjects with SLE predictably produced antibodies to a variety of nuclear antigens. A quantitative genetic association test with ANA identified genomic loci associated with high ANA phenotype. HLA was second strongest signal $\left(p=6.2 \times 10^{-6}\right)$. The frequencies of SLE risk haplotypes at several loci were significantly increased in the ANA high positive group compared to ANA negative subjects. However, SLE risk haplotypes at other loci were only high in the SLE group, suggesting their main role in a transition to clinical disease.

Conclusions The genetic risk for the development of ANA includes many of the previously documented SLE risk haplotypes. However, other genetic associations are specific for SLE, suggesting distinct risk factors for ANA and for lupus.

\section{CLINICAL USEFULNESS OF QUANTITATIVE MEASUREMENT OF ANTI-M-TYPE PHOSPHOLIPASE A2 RECEPTOR ANTIBODIES IN PATIENTS WITH MEMBRANOUS NEPHROPATHY AND COMPARISONS OF THREE QUANTITATIVE METHODS}

R Moriyama*, Y Katsumata, Y Okamoto, Y Kawaguchi, H Yamanaka. Tokyo Women's Medical University, Institute of Rheumatology, Tokyo, Japan

\subsection{6/lupus-2017-000215.284}

Background and aims Autoantibodies to M-type phospholipase A2 receptor (PLA2R) are specific markers of idiopathic membranous nephropathy (MN). It has also been suggested that anti-PLA2R antibody is associated with disease activity and prognosis but more solid evidence is needed. We aimed to establish quantitative measurement of anti-PLA2R antibodies and further investigate its clinical usefulness.

Methods Using stable cell line expressing PLA2R, we developed a quantitative cell-based enzyme-linked immunosorbent assay (ELISA) and Western blot (WB) for anti-PLA2R antibodies. The usefulness of these tests and the commercial solid phase ELISA were retrospectively studied in sera from 23 patients with biopsy-proven primary $\mathrm{MN}$, and 16 patients with lupus MN. Repeated sera were also available in 9 patients with primary MN.

Results Anti-PLA2R antibodies were detected in 12, 6, and 12 out of 23 patients with primary $\mathrm{MN}$ by the WB, the cellbased ELISA, and the commercial solid phase ELISA, respectively. Conversely, all of the samples from the lupus $\mathrm{MN}$ patients were negative. The levels of proteinuria were moderately correlated with titers of anti-PLA2R antibodies by the 3 methods ( $r=0.39$ to 0.47 ). Anti-PLA2R antibodies were significantly associated with physicians' decision on immunosuppressive therapy without prior knowledge of anti-PLA2R antibody positivity $(p<0.01)$. In all of the 6 patients who were treated with immunosuppressive therapy, titers of anti-PLA2R antibodies significantly declined by commercial solid-phase ELISA $(\mathrm{p}=0.03)$.

Conclusions This study showed that anti-PLA2R antibody is clinically useful as diagnostic and surrogate biomarkers in primary MN. In addition, the 3 methods are all reliable measurement methods for anti-PLA2R antibodies but demonstrated different performance.

\section{NAILFOLD VASCULAR FINDINGS OF ANTI-MELANOMA DIFFERENTIATION-ASSOCIATED GENE 5 ANTIBODY- POSITIVE PATIENTS WITH DERMATOMYOSITIS}

${ }^{1}$ K Komura*. ${ }^{1}$ Tsuruga Hospital, Dept of Dermatology, Tsuruga, Japan

\subsection{6/lupus-2017-000215.285}

Background and aims Rapidly progressive interstitial lung disease is complicated in majority of patients with dermatomyositis who are positive for anti-melanoma differentiationassociated gene 5 antibody (MDA5). Clinically amyopathic dermatomyositis with Heliotrope rash and Gottron's sign can offer an implication for MDA5-positivity and the critical treatment before uncovering the result of blood testing, since these patients can survive only if they received immediate and intensive therapy.We observed nailfold capillary formation of two acute patients with anti-MDA5 antibody using dermatoscopy in the current study in order to test the capability to predict the MDA5 positivity. 
Methods Nailfold of two patients with dermatomyositis were examined using dermatoscopy, (dermlite 3, 3 gen, USA.) MDA5 positivity was confirmed by ELISA.The findings were imaged using I pad mini (apple, USA). The images were compared to the patient with Jo-1, those with TIF1, or centromere.

Results Marked haemorrhages and enlarged capillaries were observed in almost all nailfolds of both hands, while only upto three nailfolds in patients with antibodies against Jo-1, TIF1, or centromere. However, loss of capillaries was not detected under the dermatoscopy at all, while these were detected in capillary scope in the literature in anti-MDA5 antibody positive patients.

Conclusions The results of the current study suggest that nailfold findings using dermatoscopy have a potential to diagnose the patients anti-MDA5 antibodies at their first visit, although there is a limitation in number of patient samples in this study. Likewise, the nailfold findings on dermatoscopy may provide visible information for the pathogensis of interstitial pneumonitis in these patients as well.

\section{IDENTIFYING EXPOSURES TO CHEMICALS IN PATIENTS WITH SLE - "A NON-TARGETED EXPOSOME APROACH"}

${ }^{1} \mathrm{C}$ Lanata*, ${ }^{2} \mathrm{~T}$ Lin, ${ }^{2} \mathrm{R}$ Gerona, ${ }^{1} \mathrm{~L}$ Criswell. ${ }^{1}$ University of California - San Francisco, Medicine, San Francisco, USA; ${ }^{2}$ University of California - San Francisco, Ob/Gyn reproductive sciences, San Francisco, USA

\subsection{6/lupus-2017-000215.286}

Background and aims Environmental exposures may play a substantial role in the pathogenesis of SLE. It recently became possible to identify and quantify a person's exposure to environmental chemicals ("the exposome") in a comprehensive fashion. This non-targeted approach has no a priori selection of chemicals. The goal of this study is to characterise multiple organic chemicals in a cohort of SLE patients and controls.

Methods Patients from the California Lupus Epidemiology Study and healthy controls were studied. Banked serum was analysed by Liquid Chromatography Quadruple Time-of-Flight Mass Spectrometry (LC-QTOF/MS). Data acquired by LCQTOF/MS includes the molecular weights of all detected parent and daughter ions, as well as retention times and peak areas. This non-targeted screening allows rapid identification of potential hits. The results of the LC-QTOF/MS analysis are matched into a database of 740 potentially detected environmental organic chemicals [EOC].

Results We present preliminary data on 19 patients with SLE and 43 controls. 193 potential EOC hits were found in patients with SLE and 417 were found in controls. In SLE patients, the number of chemicals detected per participant ranged from 34-66, with an average of 50 hit matches. Pthalates and its metabolites were the most represented chemicals, with $>50 \%$ of detected compounds in SLE. (Figure 1) Compounds of relevance include several endocrine disruptors such as Bisphenol A and Methoxyclor.

Conclusions Patients with SLE are exposed to a wide range of chemicals. LC-QTOF/MS can identify a wider range of potential chemical exposures in SLE, and aid in prioritising chemicals for further research and interventions.

\section{Environmental Organic Chemicals detected in SLE patients}

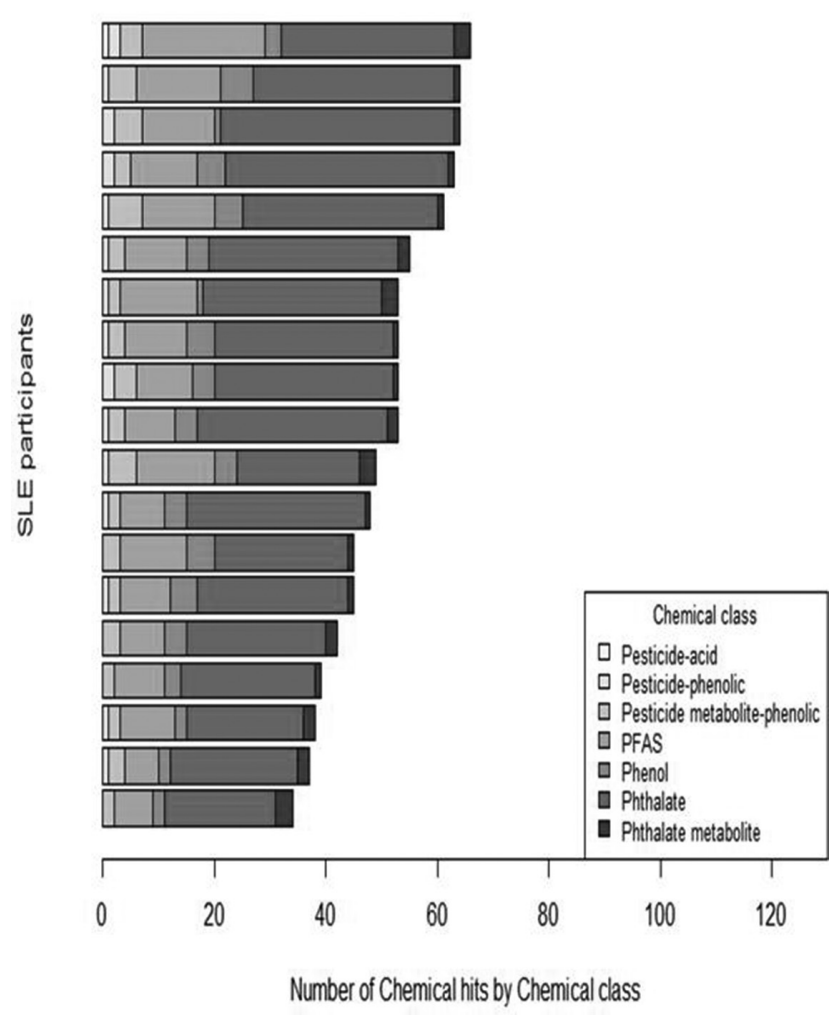

Abstract 286 Figure 1 Environmental Organic Chemicals detected in SLE patients

\section{RISK FACTORS FOR AND COMPONENTS OF METABOLIC SYNDROME: A STRUCTURAL EQUATION MODELLING ANALYSIS OF THE QUALITY OF LIFE OF PATIENTS WITH SYSTEMIC LUPUS ERYTHEMATOSUS}

SS Lee*, JW Lee, JH Kang, YR Yim, JE Kim, KE Lee, DJ Park. Chonnam Nat'I Univ Hosp, Rheumatology, Gwangju, Republic of Korea

\subsection{6/lupus-2017-000215.287}

Background and aims This study assessed: (1) the relationships among the risk factors for and components of metabolic syndrome (MetS) and health-related quality of life (HRQOL), and (2) the effects of these variables on HRQOL in a hypothesised causal model using structural equation modelling (SEM) in patients with systemic lupus erythematosus (SLE).

Methods Of the 505 SLE patients enrolled in the Korean Lupus Network (KORNET registry), 244 had sufficient data to assess the components of MetS at enrollment. Education level, monthly income, corticosteroid dose, Systemic Lupus Erythematosus Disease Activity Index, Physicians' Global Assessment, Beck Depression Inventory, MetS components, and the Short Form-36 at the time of cohort entry were determined. SEM was used to test the causal relationship based on the Analysis of Moment Structure.

Results The average age of the 244 patients was $40.7 \pm 11.8$ years. The SEM results supported the good fit of the model $\left(\chi^{2}=71.629, \mathrm{p}=0.078\right.$, RMSEA 0.034, CFI 0.972). The final 